\title{
Nursing-led management of side effects of radiation: evidence-based recommendations for practice
}

\author{
Patricia Poirier \\ University of Maine School \\ of Nursing, Orono, ME, USA
}

This article was published in the following Dove Press journal:

Nursing: Research and Reviews

22 March 2013

Number of times this article has been viewed

\begin{abstract}
It has been estimated that $50 \%-60 \%$ of patients diagnosed with cancer will receive radiation therapy at some point in their treatment. Although radiation therapy can play a significant role in the cure or control of cancer, and the palliation of symptoms, it also has side effects. Side effects of radiation therapy can interfere with patient quality of life and daily functioning. Severe side effects can lead to delays in treatment, potentially affecting the outcome of treatment. All patients receiving radiation therapy are at risk of fatigue and skin reactions in the area of the body being treated. Other side effects of radiation therapy are specific to the part of the body being treated. Radiation therapy to the head and neck area may cause oral mucositis, dryness, and nutritional deficiencies. Radiation therapy to the chest or lung area may lead to difficulty in swallowing and eating. Radiation therapy to the pelvis frequently causes diarrhea. There are many nursing interventions available to manage the side effects of treatment based on best available evidence and expert opinion. Nurses in all settings are essential in helping patients manage the side effects of treatment and maintain their quality of life. The purpose of this review is to provide nurses with evidence-based recommendations and suggestions for managing common acute side effects of radiation therapy.
\end{abstract}

Keywords: evidence-based practice, radiation therapy, side effects, nursing management

\section{Introduction}

Ms King is a 42-year-old woman ready to start a course of radiation therapy after breast-conserving surgery and chemotherapy for stage II breast cancer. Mr Lee is a 65-year-old man with locally advanced head and neck cancer undergoing concurrent chemotherapy and radiation therapy. Ms Peters is a 73-year-old woman with a long history of smoking and chronic obstructive pulmonary disease diagnosed with unresectable non-small cell lung cancer. Mr Jones is a 58-year-old African-American man who has chosen external beam radiation therapy for treatment of early-stage prostate cancer. These four patients share one common characteristic, ie, they will be receiving radiation therapy for cancer and are at risk of developing side effects from the treatment. They are all likely to need some degree of nursing intervention to help them manage their side effects and maintain their quality of life.

Nurses in radiation therapy departments apply evidence-based practice when providing patient and family education and managing side effects. ${ }^{1,2}$ However, patients receiving radiation therapy are only in the department for a short period of time each day, so much of the management of side effects of treatment falls to inpatient, primary care, or home care nurses. The purpose of this review is to provide community nurses with general recommendations for evidence-based management of common acute side effects
Correspondence: Patricia Poirier University of Maine, 5724 Dunn Hall, Orono, ME 04469 5724, USA

Email patricia.poirier@umit.maine.edu 
of radiation therapy. Nursing management of general side effects of fatigue and skin reaction and of selected site-specific side effects of radiation therapy to the chest, head and neck, and pelvis will be reviewed. Because most radiation departments have their own unique protocols, nurses should always confirm any treatment with the individual department.

Radiation therapy is the use of ionizing radiation for the purpose of control or cure of cancer or the palliation of symptoms due to advanced cancer. Ionizing radiation causes damage to the DNA of cells, limiting their ability to divide and reproduce. ${ }^{3}$ Radiation causes damage to both cancerous and normal cells. Given nonlethal doses and adequate recovery time, normal cells are generally able to repair and regenerate. Side effects of treatment, which are a result of damage to normal cells, may impact quality of life for patients and their ability to carry out their usual activities. In severe cases, side effects may lead to hospitalization and possible delays in treatment.

Modern radiation therapy techniques aim to pinpoint the dose directly at the target site with the goal of minimizing side effects. Nursing assessment and management can help to minimize further the impact of side effects on quality of life for patients.

Radiation therapy may be delivered in a variety of ways, including intraoperative radiation and radioactive implants. The most common method of delivery is external beam radiation delivered by a linear accelerator. Using this type of treatment, patients receive a daily dose or fraction (measured in Gy or cGy) of radiation 5 days a week for a period of several weeks. Daily and cumulative doses vary by disease, treatment site, and purpose of treatment. ${ }^{3}$

Side effects of radiation depend on treatment site, daily dose, concurrent chemotherapy, and individual patient characteristics (Table 1). Side effects of radiation therapy are specific to the part of the body being treated, and generally begin after approximately 2 weeks of treatment or at a cumulative dose of 2000 cGy. $^{3}$ Side effects increase in severity as treatment progresses, and then gradually subside after treatment is completed. Skin reactions and fatigue may occur in any patient receiving radiation therapy.

Much of the nursing care of patients experiencing side effects from radiation therapy is based on tradition and provider preference rather than actual evidence. The increased complexity of patient care and demands from regulatory agencies require nursing practice to be based on the best available evidence. Multiple definitions of evidence-based practice exist. Boswell and Cannon's definition of evidencebased practice as "a process of using confirmed evidence (research and quality improvement), decision-making, and nursing expertise to guide the delivery of holistic patient care"4 directed this review, which discusses the evidence where available, using the Oncology Nursing Society (ONS) Putting Evidence into Practice (PEP) framework. The ONS PEP resource identifies six levels of recommendations for patient care and teaching on a variety of topics (http://www. ons.org/Research/PEP). These categories are:

- Recommended for practice: interventions for which effectiveness has been demonstrated by strong evidence from rigorously designed studies, meta-analysis, or systematic reviews, and for which expectation of harm is small compared with the benefits.

- Likely to be effective: interventions for which effectiveness has been demonstrated from a single rigorously conducted controlled trial, consistent supportive evidence from well designed controlled trials using small samples, or guidelines developed from evidence and supported by expert opinion.

- Benefits balanced with harm: interventions for which clinicians and patients should weigh the beneficial and harmful effects according to individual circumstances and priorities.

Table I Side effects of radiation therapy by treatment site*

\begin{tabular}{|c|c|c|c|c|c|c|}
\hline & Brain & Head and neck & Breast & Chest & Abdomen or pelvis & Prostate \\
\hline Hair loss (in area of body being treated) & $\checkmark$ & $\checkmark$ & & $\checkmark$ & $\checkmark$ & $\checkmark$ \\
\hline Skin changes (in part of body being treated) & $\checkmark$ & $\checkmark$ & $\checkmark$ & $\checkmark$ & $\checkmark$ & $\checkmark$ \\
\hline Fatigue & $\checkmark$ & $\checkmark$ & $\checkmark$ & $\checkmark$ & $\checkmark$ & $\checkmark$ \\
\hline Diarrhea & & & & & $\checkmark$ & $\checkmark$ \\
\hline Nausea and vomiting & $\checkmark$ & & & & $\checkmark$ & \\
\hline Mouth changes: mucositis, xerostomia & & $\checkmark$ & & & & \\
\hline Esophagitis & & & & $\checkmark$ & & \\
\hline Urinary and bladder changes & & & & & $\checkmark$ & $\checkmark$ \\
\hline Other side effects & $\begin{array}{l}\text { Headache, } \\
\text { blurry vision }\end{array}$ & Taste changes & $\begin{array}{l}\text { Tenderness } \\
\text { or swelling }\end{array}$ & $\begin{array}{l}\text { Cough, shortness } \\
\text { of breath }\end{array}$ & & \\
\hline
\end{tabular}

*Adapted from NIH Publication No $12-7157$ Radiation Therapy and You. ${ }^{54}$ 
- Effectiveness not established: interventions for which insufficient or conflicting data or data of inadequate quality currently exist, with no clear indication of harm.

- Unlikely to be effective: interventions for which lack of effectiveness has been demonstrated by negative evidence from a single rigorously conducted controlled trial, consistent negative evidence from well designed controlled trials using small samples, or guidelines developed from evidence and supported by expert opinion.

- Not recommended for practice: interventions for which lack of effectiveness or harm has been demonstrated by strong evidence from rigorously conducted studies, metaanalyses, or systematic reviews, or interventions where the costs, burden, or harm associated with the intervention exceed the anticipated benefit.

\section{Side effects common to all sites Skin reactions}

Radiation skin reactions or radiation dermatitis occur in nearly all patients receiving radiation therapy. Early radiation therapy equipment delivered the majority of the dose to the surface of the skin. Therapeutic and maximum tolerated doses were determined by the degree of erythema present. Modern equipment and sophisticated treatment planning techniques produce a "skin-sparing" effect, with the therapeutic dose delivered to the target organ, minimizing the dose to the skin. Still, approximately $95 \%$ of patients receiving radiation therapy may experience some degree of skin reaction, ranging from mild erythema to more serious and uncomfortable moist desquamation. ${ }^{5}$ Severe moist desquamation results in pain and discomfort, and may require a break in treatment, possibly compromising the effectiveness of treatment. Hair loss may also occur at the treatment area, and may be permanent at high doses of radiation (6000 cGy or greater). ${ }^{1}$ It is important to note that radiation dermatitis and hair loss only occur within the area of the body being treated. Thus, patients can be reassured that hair loss on the head is not expected from radiation unless the brain or scalp is being treated.

Many factors contribute to the degree of skin reaction that an individual patient may experience. Treatment-related factors, such as type of energy (eg, photon versus electron), daily dose, size of the treatment field, and use of bolus material (the purpose of which is to increase the dose to the skin surface) affect the degree of skin reaction. Individual patient characteristics also play a role in the development of skin reactions. These may include obesity with resulting skin folds, genetic factors, comorbid conditions such as diabetes, nutritional status, age, race, and ethnicity, smoking history, medications, concurrent chemotherapy, sun exposure, and mobility. ${ }^{6}$ Specific diseases, such as skin cancer and inflammatory breast cancer treated with radiation therapy, by their nature require a significant skin dose and are expected to have an intense skin reaction. Thus, the overall goal of nursing management for radiation skin reactions is to delay and/or reduce their intensity, not eliminate them altogether. ${ }^{7}$

A key element in the management of radiation skin reactions is routine assessment using a standardized assessment tool. ${ }^{5}$ Skin assessment should be completed at baseline prior to initiation of treatment and at a minimum of once a week. The expansion of electronic medical records allows for standardization and tracking of skin integrity.

After approximately 2 weeks of treatment or a dose of $2000 \mathrm{cGy}$, patients may develop mild to moderate erythema. The skin in the treatment area may be reddened, edematous, and feel warm to touch. Dry desquamation, characterized by pruritus and dry flaking skin, may develop after doses of 3000 cGy. Moist desquamation occurs at doses greater than 4000 cGy. Serous drainage may be present in areas of friction, such as inframammary folds and the axilla. ${ }^{8,9}$ In severe cases of moist desquamation, patients may have pain, bleeding, infection, and/or require a break in treatment. The majority of acute skin reactions resolve within 1-2 weeks following completion of treatment. Patients should be instructed to protect the treated skin indefinitely from sun and excessive cold.

There is currently no standard for skin care during radiation therapy. Most radiation therapy departments have their own skin care protocols for patients to follow during treatment. In the past, patients were discouraged from washing the skin in the treatment area or using a deodorant if the axilla was in the treatment field. This can cause distress for patients ${ }^{10}$ and is not supported by the literature. ${ }^{5}$ Therefore, allowing patients to continue their usual personal hygiene measures during treatment is now recommended in practice. The treatment area may be gently washed with mild soap or a $\mathrm{pH}$-neutral cleanser and water. Patients should pat the area dry using a soft towel, and should ensure that any skin folds in the treatment area are completely dry. Patients receiving cranial radiation can wash with mild shampoo, such as baby shampoo.

A deodorant may be used as long as the skin is intact. Practitioners generally assume that use of deodorant with aluminum would act as a bolus and increase the skin dose. Use or nonuse of deodorant has not been widely studied, but a nonclinical study by researchers found no differences 
in skin surface dose with aluminum versus nonaluminum products. ${ }^{11}$ A study of 84 women receiving breast radiation found that axillary skin reactions were less frequent in women who used a nonaluminum deodorant compared with women who used no deodorant. ${ }^{12}$ If shaving is necessary, an electric razor should be used.

Effectiveness has not been established for a wide variety of topical skin care products because there is no convincing literature to either support or refute their use during radiation therapy. ${ }^{5}$ Therefore, patients may continue to use moisturizing creams or lotions of their choice as long as the skin is intact. Patients should use products specifically recommended by their radiation therapy practitioner. Patients do not need to avoid topical agents for any set period of time prior to treatment, but should not apply them immediately before treatment. ${ }^{13}$ Calendula ointment has been found likely to be effective and thus may be recommended for women receiving breast irradiation. ${ }^{14,15}$

The skin in the treatment area should be protected from irritants and extremes of hot or cold. Patients should wear loose fitting clothing, avoid tape or adhesives, avoid ice or heating pads, use sunscreen of at least factor 30, and protect any exposed skin from the cold. Patients should avoid swimming in lakes or pools and using hot tubs or saunas if the treated skin is irritated. ${ }^{5}$

Moisturizers and sparing use of topical corticosteroids may provide comfort for patients who develop pruritus from dry desquamation. Dressings may be used for moist desquamation to contain bleeding, exudates, and drainage. Antimicrobials (either topical or oral) may be used if infection is present. ${ }^{5}$ Topical hyaluronic acid cream has been found likely to be effective and may be used to manage moist desquamation in the absence of infection. ${ }^{5}$

In summary, some degree of skin irritation is likely in the majority of patients receiving external beam radiation. Routine assessment using a standardized tool is key to managing skin reactions. Patients may continue their usual skin care practices using products recommended by their practitioner. The skin in the treatment area should be protected from tight clothing and heat and cold. Nurses are essential in helping patients manage skin reactions, remain comfortable, and maintain usual activities. Research is needed to establish a standard of care for management of radiation skin reactions because the literature provides no clear direction. McQuestion suggests that future research may need to look at the underlying physiologic mechanism of radiation damage because no product is likely to be able to prevent the damage. ${ }^{15}$ Agency-specific protocols may be developed using available evidence, expert opinion and consensus, and patient and provider preference. ${ }^{16}$ Ease of use and cost also need to be considered when making treatment recommendations.

\section{Fatigue}

Fatigue continues to be one of the most distressing side effects reported by patients, occurring in 65\%-100\% of patients receiving radiation therapy. ${ }^{17,18}$ Cancer-related fatigue is subjective and multidimensional, with physical, psychological, social, and spiritual aspects. ${ }^{19}$ Radiation therapy-related fatigue follows a predictable pattern, beginning in approximately the second week of treatment, increasing as treatment progresses, peaking at the end of treatment, and returning to near baseline by the one-month follow-up visit. ${ }^{1,20}$ Both the $\mathrm{ONS}^{21}$ and the National Comprehensive Cancer Network (NCCN) ${ }^{22}$ have published extensive guidelines on the management of cancer-related fatigue.

As with all treatment-related side effects, routine assessment is key to managing radiation therapy-related fatigue. There are many valid and reliable instruments to measure either a single dimension of fatigue or its multidimensional nature. These instruments include the Piper Fatigue Scale, the Brief Fatigue Inventory, and the Functional Assessment of Cancer Therapy-Fatigue. ${ }^{23}$ The NCCN recommends that a single-item screening be conducted at each visit: "How would you rate your fatigue on a scale of $0-10$ over the past 7 days?"22 Butt et al suggest that single screening can lead to improved symptom control, objective functional status, and overall quality of life. ${ }^{24}$

A primary evaluation of fatigue is indicated for adult patients who rate their fatigue on the single item measure as either moderate (rating of 4-6) or severe (rating of 7-10). ${ }^{22}$ This primary evaluation should include an assessment of disease status to rule out progression, an assessment of treatable contributing factors, an assessment of social support status/ availability of caregivers, and an indepth fatigue history. ${ }^{22}$ The fatigue history should assess onset, pattern, and duration of fatigue, change in fatigue over time, associated or alleviating factors, and interference with function. ${ }^{22}$ Treatable contributing factors include pain, emotional distress, anemia, sleep disturbances, nutritional deficit/imbalances, decreased functional status, and medical comorbidities such as hypothyroidism. ${ }^{22}$ In a systematic review and meta-analysis of correlates of cancer-related fatigue, Oh and Seo found that all symptoms (pain, nausea and vomiting, dyspnea, lack of appetite) and psychological distress (depression and anxiety) were significantly correlated with cancer-related fatigue. ${ }^{25}$ 
The implication for nursing is that treatment of these related factors can reduce the severity of cancer-related fatigue and its resultant distress.

All patients and families should receive fatigue-related education and counseling, including information about the pattern of fatigue related to radiation therapy. Patients should be reassured that fatigue is to be expected, and is not an indicator of disease progression. ${ }^{22}$ The NCCN guidelines provide general strategies that can be recommended to all patients, including self-monitoring of fatigue levels, energy conservation (set priorities and realistic expectations, pace activities and schedule them for times of peak energy, postpone nonessential activities, delegate where possible, limit naps to less than one hour per day, maintain a structured daily routine), and use of distraction. ${ }^{22}$ The ONS PEP guidelines identify these interventions as likely to be effective. ${ }^{21}$

Both the ONS PEP guidelines and the NCCN guidelines recommend activity enhancement or exercise for prevention and management of cancer-related fatigue. ${ }^{21,22}$ Exercise has been the only intervention for cancer-related fatigue to show consistent beneficial effects on not only fatigue but also on overall quality of life. ${ }^{19,26}$ Most studies of the impact of exercise on cancer-related fatigue have been conducted in women with breast cancer. ${ }^{26}$ There are studies that suggest exercise may also benefit patients with lung, head and neck, prostate, or colorectal cancer. ${ }^{26-31}$ Home-based exercise programs are feasible and effective for cancer-related fatigue. The challenge for nurses is finding ways to help patients maintain an exercise program. Wanchai et al found that provision of written materials along with a step pedometer and telephonebased support were effective interventions in encouraging patients to maintain their physical activity. ${ }^{19}$ Patients need to be carefully screened prior to initiating any exercise routine. The exact type and duration of exercise for cancer-related fatigue has not been established. Exercise routines should be tailored to the individual patient.

Other nonpharmacologic interventions recommended by the NCCN and deemed either likely to be effective or effectiveness not established by the ONS include: physically and energy-based therapies, such as reiki and massage; psychosocial interventions such as cognitive behavioral therapy, individual or group psychotherapy, and mindfulnessbased therapies; music and art therapy; and nutritional consultation. $^{22,32}$ Pharmacologic agents to treat cancerrelated fatigue have not been shown to have much benefit, although the NCCN guidelines suggest that psychostimulants may be considered when other treatable causes have been eliminated. ${ }^{22}$ Use of erythropoietin-stimulating agents may be considered for cases of significant anemia after balancing their potential risks and benefits. ${ }^{21,32}$

Nursing management of radiation therapy-related fatigue then would include routine assessment of fatigue, patient and family education about fatigue, and encouraging activity when not contraindicated. Nurses can support patients in setting priorities and promoting energy conservation when experiencing fatigue.

\section{Site-specific side effects Head and neck area}

Radiation therapy to the head and neck area presents a major challenge to patients and nurses. Side effects are often severe and disabling, and may significantly impact quality of life. The lived experience of radiation therapy to the head and neck area profoundly affects activities of daily living, requiring nursing support and education throughout the trajectory of treatment. ${ }^{33}$ Many patients with head and neck cancers receive concurrent chemotherapy, which may lead to earlier development and increased severity of side effects. Side effects of radiation therapy to the head and neck area include skin reactions, oral mucositis resulting in pain and nutritional deficiencies, taste changes further impacting nutritional status, and mouth dryness or xerostomia. Radiation therapy-related side effects to the head and neck area begin after approximately 2 weeks of treatment and increase in severity over the course of the treatment. Patients receiving concurrent chemotherapy may have earlier onset and greater severity of side effects. Most acute side effects resolve within 2-4 weeks after completing radiation therapy. Nursing care is essential in minimizing these side effects and in particular in supporting nutritional status.

\section{Oral mucositis}

Mucositis is an inflammatory response of mucosal epithelial cells to the cytotoxic effects of chemotherapy or radiation therapy. ${ }^{34}$ Oral mucositis disrupts the integrity of the oral cavity and may be associated with decline in functional status and quality of life, pain, malnutrition, and infection. ${ }^{25,34}$ Severe oral mucositis may result in treatment delays, which may impact the overall treatment outcome. ${ }^{34}$ This is particularly significant in head and neck cancers because unplanned breaks in treatment have been shown to impact both local control and overall survival. ${ }^{36}$ Russo et al report that local control may be reduced by as much as $1 \%$ for each day that radiation is interrupted. ${ }^{36}$ Nursing management is essential in reducing unplanned treatment breaks. As with most radiation therapy-related side effects, oral mucositis generally 
begins within 2 weeks of treatment, increases in severity as treatment progresses, and resolves within 1-2 weeks after treatment is stopped. Many patients with cancers of the head and neck region receive concurrent chemotherapy, so are at increased risk of earlier onset, greater severity, and longer duration of oral mucositis. ${ }^{1}$

Assessment is the first step in managing oral mucositis. Assessment should be performed routinely using a standardized assessment tool and should assess function, pain, and integrity of the oral mucosa. ${ }^{34}$ Ideally, patients receiving treatment for head and neck cancers should see their dentist prior to initiating treatment and regularly throughout treatment. ${ }^{37}$ Fluoride rinses are frequently recommended for patients receiving radiation therapy to the head and neck area. ${ }^{37,38}$

Routine oral care is recommended for all patients receiving radiation therapy to the head and neck. Oral care does not prevent mucositis, but it can reduce the duration and severity of mucositis. ${ }^{39,40}$ Oral care can reduce pain and bleeding, prevent infection, and minimize future dental complications. ${ }^{34,40,41}$ The literature does not indicate exactly what agents should be used in an oral care protocol, but rather supports the principle that the protocol should be standardized and easy for patients to follow and adhere to. ${ }^{34,42}$

Patients should brush their teeth with an ultrasoft toothbrush twice daily as long as it is comfortable to do so. Flossing should be performed at least once a day or as advised by their practitioner. If patients find the use of a toothbrush painful, they may use gauze or foam swab dipped in rinsing solution. ${ }^{43}$ Patients should rinse their mouth 4-6 times daily with a bland rinse at the beginning of treatment and increase the frequency to every 1-2 hours when symptoms develop. Bland rinses have not been supported by the literature, but are recommended by expert opinion. Bland rinses include normal saline, baking soda, or a mixture of saline and baking soda. Patients can make their own rinses using a teaspoon of salt or baking soda per pint of water. Rinses may be administered at room temperature or refrigerated according to patient preference. Many departments recommend multiagent rinses ("Magic" or "Miracle"). These rinses typically contain lidocaine, diphenhydramine, and Maalox. Albeit anecdotally, patients have reported benefit from these rinses, but research has not supported their superiority over bland rinses. They tend to be more expensive than bland rinses, and there is concern that the numbing effect of the lidocaine may create a potential for injury, so there is no reason to recommend these multiagent rinses over bland rinses. ${ }^{34}$ Patients should avoid tobacco, alcohol, and acidic, hot, spicy, or rough foods throughout treatment.
Nurses should assess patients for the presence of any infection, especially yeast infections, and these should be treated as necessary. There is some suggestion that use of antimicrobial agents might delay the onset or reduce the severity of mucositis in patients receiving radiation therapy, but the evidence is not strong enough to recommend their widespread use. ${ }^{34,44}$ Patients should be assessed for pain and treated as appropriate. Oral mucositis may require opioid analgesics. Transdermal opioids may be required if patients are unable to take medications by mouth. ${ }^{38}$

\section{Xerostomia}

Radiation-induced xerostomia encompasses both the subjective sensation of oral dryness along with an objective decrease in the production of saliva. ${ }^{45}$ Radiation-induced xerostomia is a direct result of destruction of salivary acini and is dependent on the amount of salivary gland tissue in the treatment field and the total dose delivered. ${ }^{46}$ Unilateral versus bilateral salivary gland irradiation also impacts the severity and duration of xerostomia. Severe dysfunction of the salivary glands occurs with radiation doses above 5200 cGy. Given that most patients with head and neck cancers generally receive radiation doses of 6000-7000 cGy, chronic xerostomia is common. ${ }^{46}$ Depending on the dose to the salivary glands, some return of salivary function may occur over time. In a small sample of patients postradiation to the head and neck region, Braam et al found that xerostomia-related quality of life improved over time, although $41 \%$ of patients complained of moderate to severe dryness at 5-year follow-up. ${ }^{47}$

Advanced treatment techniques, such as intensitymodulated radiation therapy, three-dimensional conformal radiation therapy, image-guided radiation therapy, and proton therapy may reduce the incidence and severity of side effects, including xerostomia. ${ }^{48}$ However, these modalities are expensive, require highly trained personnel, and are still not in widespread use for all patients with head and neck cancers. Radioprotectant medications, such as amifostine, have shown promise in prevention of xerostomia in patients receiving head and neck radiation and may be considered to decrease acute and late xerostomia. ${ }^{49,50}$ These medications are costly, require subcutaneous or intravenous administration, and have significant side effects. Therefore, use of radioprotectants has not become standard of care.

Nursing management of radiation-induced xerostomia falls into one of two categories, ie, saliva stimulants and saliva substitutes. Saliva stimulants or sialogogues may be used concomitantly with radiation or used after radiation. ${ }^{51}$ 
Pilocarpine, a nonspecific exocrine gland stimulant, is the most widely used sialogogue for radiation-induced xerostomia. ${ }^{51,52}$ The side effects of pilocarpine include sweating, flushing, rhinitis, and increased bowel and bladder motility, so patient acceptance of its use may be limited. ${ }^{38,45,52}$ Nurses can play a role in increasing adherence by monitoring use of these medications by patients and reinforcing their benefit.

Saliva substitutes are widely available without a prescription and are well tolerated. These substitutes provide shortterm relief and comfort. Available products include Biotene ${ }^{\circledR}$ (GlaxoSmithKline, Mississauga, ON, Canada) and Mouth Kote $^{\circledR}$ (Parnell Pharmaceuticals, San Rafael, CA, USA). Mucoadherents such as MuGard ${ }^{\circledR}$ (MuGard, Dallas, TX, USA) have been shown to reduce pain from stomatitis. ${ }^{53}$ Frequent oral rinsing, sipping on ice water or ice chips, and sucking on sugar-free hard candies may also provide some relief from xerostomia. ${ }^{43,54}$

There is growing evidence that acupuncture may be beneficial for radiation-induced xerostomia. ${ }^{55-57}$ Given that integrative medicine is becoming more mainstream, nurses can suggest complementary and alternative therapies as additional options to help patients manage their symptoms.

\section{Nutritional deficiencies}

Patients receiving radiation therapy to the head and neck region, particularly those receiving concurrent chemotherapy and radiation therapy, are at high risk of nutritional deficiencies. Many of these patients present with malnutrition. ${ }^{38}$ Pain and swallowing difficulties combined with oral dryness and taste changes from radiation contribute further to the problem. Difficulty eating as a result of radiation may lead to loss of social interaction and increasing social isolation because patients may be insecure and embarrassed about eating in the presence of others. ${ }^{33}$ Nursing assessment and interventions are vital to supporting patients' nutritional status and quality of life during treatment. Patients should be weighed at the minimum of once a week. Hydration status should also be evaluated. Extremely high-risk patients may have a feeding tube placed prior to initiation of treatment. ${ }^{38}$ Nurses can provide teaching regarding tube feedings and tube maintenance. Nutritional consultation with a registered dietitian has been shown to improve nutritional status and maintain quality of life in patients receiving radiation therapy. ${ }^{58,59}$

Dietary modifications may help patients maintain their nutritional status during treatment. Patients may find small frequent meals and snacks easier than three large meals. Foods should be high in protein and calories. Soft, bland, and moist foods are easier to swallow. These would include cream soups, cooked cereals, mashed potatoes, scrambled eggs, puddings, custards, yogurts, instant breakfast shakes, and high protein supplements. ${ }^{54}$ Powdered milk may be added to foods to increase protein and calories. There are many high protein supplements available, such as Boost, Ensure, and Carnation Instant Breakfast. Supplements may be covered by insurance if they are the sole source of nutrition. Foods that are warm (not hot) or room temperature may be easier to tolerate. Patients should be encouraged to take small bites, chew slowly, and sip liquids with their meals. Foods moistened with gravy, sauce, broths, or yogurts may help when patients develop xerostomia. ${ }^{54}$ Patients should avoid extremely hot foods or drinks, spicy foods, foods that are acidic such as tomatoes and citrus, and sharp or crunchy foods such as potato chips. ${ }^{54}$ Smoking and alcohol should also be avoided.

Nursing care is essential in helping patients manage oral mucositis and xerostomia. Nursing assessment and intervention in collaboration with a multidisciplinary team consisting of dentists and dietitians can help maintain nutritional status and prevent complications.

\section{Chest/lung}

The major side effect of radiation for lung or esophageal cancer requiring nursing intervention is esophagitis. The etiology and timing of esophagitis is similar to that of oral mucositis. ${ }^{34}$ Esophagitis can lead to pain, difficulty swallowing, and ultimately nutritional deficiencies. Patients at highest risk for development of esophagitis include those receiving concurrent chemotherapy and those receiving high doses of radiation to a large treatment area.

Dietary modification is the major nursing intervention for esophagitis. A soft, bland, high-calorie, and high-protein diet should be provided. High-protein supplements may be used. Patients should avoid tobacco and alcohol products and foods that are spicy, acidic, or crunchy. Patients should be encouraged to eat slowly, cut foods into small pieces, and chew thoroughly. Using a straw to sip fluids, sitting upright and bending head slightly forward when eating or drinking, and sitting or standing for 30 minutes after eating may reduce reflux. ${ }^{54,60}$

Pharmacologic interventions to manage esophagitis include antacids taken before and after meals, proton pump inhibitors for symptomatic relief, and promotility agents as an adjunct to acid suppression. ${ }^{60,61}$ Multiagent products such as "Magic" or "Miracle" mouthwashes containing topical anesthetics may also be used. ${ }^{59}$ Nursing assessment of pain 
is important, and nonsteroidal or opioid analgesics may be required. ${ }^{60}$

\section{Pelvis}

Side effects of radiation therapy to the abdomen and pelvis depend on the amount of stomach and/or bowel and bladder that is in the treatment field. Side effects may include nausea and vomiting, radiation cystitis, and diarrhea. Diarrhea is the most frequently reported and distressing side effect of pelvic or abdominal radiation, and occurs in more than $80 \%$ of patients. ${ }^{62}$

Radiation therapy for cervical, ovarian, prostate, or colorectal cancer inevitably involves some dose of radiation to the gut. This results in disruption of the normal bacteria flora, leading to diarrhea. ${ }^{63}$ Diarrhea is difficult to define. The ONS PEP team defined diarrhea as an "abnormal increase in liquidity in stool and stool frequency greater than or equal to four to six over baseline with or without nocturnal bowel movements that may be accompanied by abdominal cramping". ${ }^{32}$ Nurses need to assess patients with diarrhea for signs of dehydration or perirectal skin irritation. Diarrhea generally begins after approximately 2 weeks of treatment and continues throughout the course of treatment. The small bowel is more sensitive to radiation than is the colon or rectum. Therefore, the degree of small bowel in the treatment field influences the degree of diarrhea. Patients receiving radiation to large pelvic and/or abdominal areas are at greatest risk for developing watery diarrhea and cramping. Patients receiving radiation to smaller pelvic fields such as for prostate cancer are more likely to experience frequent soft bowel movements rather than pronounced diarrhea. ${ }^{1}$ Depending on the amount of bowel treated and the dose received, patients may continue to have bowel irritability after treatments are completed.

Dietary management of radiation-induced diarrhea remains somewhat controversial, with some practitioners recommending low-fiber diets and others recommending high-fiber diets. Most current expert opinion recommends diets that are high in soluble fiber and low in insoluble fiber. ${ }^{32}$ Foods that are high in insoluble fiber should be avoided, and patients should avoid raw fruits and vegetables, whole grain breads, nuts, popcorn, skins, seeds, and legumes during radiation therapy to the pelvis or abdomen. Foods such as apple sauce, oatmeal, bananas, cooked carrots, cream of wheat, and noodles are allowed. Spicy, greasy, fatty, or fried foods, milk products, and alcohol should be avoided. Patients should increase their fluid intake but limit caffeine.

Antidiarrheal medications, such as loperamide or diphenoxylate, are likely to be effective for mild diarrhea. ${ }^{64}$ Psyllium fiber supplementation (Metamucil ${ }^{\circledR}$, Procter and Gamble,
Table 2 Nursing interventions for common side effects of radiation therapy

\begin{tabular}{|c|c|}
\hline Side effect & Nursing intervention \\
\hline \multirow[t]{9}{*}{ Skin changes } & Routine assessment \\
\hline & Patient teaching \\
\hline & Wash gently with mild soap and water \\
\hline & Pat skin dry \\
\hline & Use lotions or creams as recommended \\
\hline & Protect skin from heat and cold \\
\hline & Avoid tight clothing \\
\hline & May use deodorant if skin intact \\
\hline & $\begin{array}{l}\text { Use topical agents/dressings if needed for moist } \\
\text { desquamation }\end{array}$ \\
\hline \multirow[t]{7}{*}{ Fatigue } & Routine assessment \\
\hline & Patient teaching and support \\
\hline & Expected pattern of fatigue \\
\hline & Maintain exercise routine \\
\hline & Practice energy conservation techniques, distraction \\
\hline & $\begin{array}{l}\text { Consider complementary therapies: reiki, massage, } \\
\text { mind-body therapies }\end{array}$ \\
\hline & Monitor impact on daily activities \\
\hline \multirow[t]{16}{*}{$\begin{array}{l}\text { Mouth } \\
\text { changes }\end{array}$} & $\begin{array}{l}\text { Routine assessment including weight and integrity of } \\
\text { oral mucosa }\end{array}$ \\
\hline & Patient teaching and support \\
\hline & Quit smoking \\
\hline & Follow oral care protocol including bland rinses \\
\hline & High protein, high calorie diet \\
\hline & Soft, bland, moist diet \\
\hline & Oral supplements as needed \\
\hline & Avoid alcohol, spicy or acidic foods \\
\hline & Saliva substitutes \\
\hline & Monitor/maintain tube feedings if present \\
\hline & Monitor/reinforce pharmaceutical interventions \\
\hline & including saliva stimulants \\
\hline & Assess and manage pain \\
\hline & Monitor nutritional and hydration status \\
\hline & Consider referral to dentist and/or dietitian \\
\hline & Consider acupuncture for xerostomia \\
\hline \multirow[t]{13}{*}{ Esophagitis } & Routine assessment \\
\hline & Patient teaching \\
\hline & High protein, high calorie diet \\
\hline & Soft, bland diet \\
\hline & Sit or stand for minimum of 30 minutes after eating \\
\hline & Small frequent meals \\
\hline & Oral supplements as needed \\
\hline & Avoid alcohol, spicy or acidic foods \\
\hline & Antacids before or after meals for comfort \\
\hline & Monitor/reinforce pharmaceutical interventions \\
\hline & Assess and manage pain \\
\hline & Monitor nutritional and hydration status \\
\hline & Consider referral to dietitian \\
\hline \multirow[t]{10}{*}{ Diarrhea } & Routine assessment including integrity of peri-rectal area \\
\hline & Patient teaching \\
\hline & Diet low in insoluble fiber; high in soluble fiber \\
\hline & Increase fluids, limit caffeine \\
\hline & Avoid fatty, fried foods, milk products, alcohol \\
\hline & Consider psyllium fiber supplements \\
\hline & Consider probiotics if not contraindicated \\
\hline & Monitor/reinforce pharmaceutical interventions \\
\hline & including antidiarrheal medications \\
\hline & Monitor hydration status \\
\hline
\end{tabular}


Cincinnati, OH, USA) is often used to increase soluble fiber. There is increasing evidence that the prophylactic use of probiotics such as VSL \#3 and Lactobacillus casei DN-114 001 can reduce the incidence and severity of radiation-induced diarrhea. ${ }^{63}$ Probiotics contain live micro-organisms that can alter host microflora and restore the balance between proinflammatory and anti-inflammatory cytokines. ${ }^{63,65-67}$ Probiotics are generally safe, but because they contain viable microorganisms, they should be used in caution in patients who are immunocompromised. ${ }^{63}$ Recommendations for use of probiotics need to be based on availability of reliable products. ${ }^{65}$

\section{Conclusion}

Patients receiving radiation therapy experience a variety of side effects that may impact their overall quality of life (Table 2). Nurses in all settings who encounter patients receiving radiation therapy are key to assessing for the impact of side effects on patients' lives and providing targeted education and recommendations for self-management, including use of complementary and alternative therapies. Nurses in the community caring for patients receiving radiation therapy can identify patients at high risk for development of side effects, reinforce interventions recommended by radiation practitioners, and evaluate the effectiveness of those interventions. Management of side effects of treatment not only leads to improved quality of life for patients, but also may lead to improved treatment outcomes and better overall survival.

\section{Disclosure}

The authors report no conflicts of interest in this work.

\section{References}

1. Moore-Higgs GJ, Watkins-Bruner D, Balmer L, et al. The role of licensed nursing personnel in radiation oncology. Part A: results of a descriptive study. Oncol Nurs Forum. 2003; 30(1): 51-58.

2. Moore-Higgs GJ, Watkins-Bruner D., Balmer L, et al. The role of licensed nursing personnel in radiation oncology. Part B: Integrating the ambulatory care nursing framework. Oncol Nurs Forum. 2003; 30(1): 59-64.

3. Bruner DW, Haas ML, Gosselin-Acomb TK. Radiation Oncology Nursing Practice and Education. 3rd e. Pittsburgh, PA: Oncology Nursing Society; 2006.

4. Boswell C, Cannon S. Introduction to Nursing Research: Incorporating Evidence Based Practice, 3rd ed. Burlington, MA: Jones and Bartlett; 2014.

5. Feight D, Baney T, Bruce S, McQuestion M. Putting evidence into practice: evidence-based interventions for radiation dermatitis. Clin J Oncol Nurs. 2011;15(5):481-492.

6. Ryan JL, Bole C, Hickok JT, et al. Post-treatment skin reactions reported by cancer patients differ by race, not by treatment or expectations. $\mathrm{Br} \mathrm{J}$ Cancer. 2007;97(1):14-21.

7. Primavera G, Carrera M, Berardesca E, Pinnaró P, Messina M, Arcangeli G. A double-blind, vehicle-controlled clinical study to evaluate the effectiveness of MAS065D (XClair), a hyaluronic acid-based formulation, in the management of radiation-induced dermatitis. Cutan Ocul Toxicol. 2006:25(3):165-171.
8. Baney T, McQuestion M, Bell K, et al. Radiodermatitis. In: Eaton LH, Tipton JM, Irwin M, editors. Putting Evidence into Practice: Improving Oncology Patient Outcomes. Pittsburgh, PA: Oncology Nursing Society; 2011.

9. Sparks S. Radiodermatitis. In: Hass M, Hogie W, Moore-Higgs G, Gosselin-Acomb TK, editors. Radiation Therapy: A Guide to Patient Care. St Louis, MO: Mosby Elsevier; 2007.

10. McQuestion M. Radiation-induced skin reactions. In: Haas ML, MooreHiggs GL, editors. Principles of Skin Care and the Oncology Patient. Pittsburgh, PA: Oncology Nursing Society; 2010.

11. Burch SE, Parker SA, Vann AM, Arazie JC. Measurement of 6_MV $\mathrm{X}$-ray surface dose when topical agents are applied prior to external beam irradiation. Int J Radiat Oncol Biol Phys. 1997:38(2): $447-451$.

12. Theberge V, Harel F, Dagnault A. Use of axillary deodorant and effect on acute skin toxicity during radiotherapy for breast cancer: a prospective randomized noninferiority trial. Int J Radiat Oncol Biol Phys. 2009;75(4):1048-1052.

13. Bieck T, Phillips S. Appraising the evidence for avoiding lotions or topical agents prior to radiation therapy. Clin J Oncol Nurs. 2011;14(1): 103-105.

14. Pommier P, Gomez R, Sunyach MP, D'Hombres A, Carrie C, Montbarbon X. Phase III randomized trial of Calendula officinalis compared with trolamine for the prevention of acute dermatitis during irradiation for breast cancer. J Clin Oncol. 2004;22(8):1447-1453.

15. McQuestion M. Evidence-based skin care management in radiation therapy: clinical update. Semin Oncol Nurs. 211;27(2):e1-e17.

16. Bergstrom K. Development of a radiation skin care protocol and algorithm using the Iowa Model of Evidence-Based Practice. Clin J Oncol Nurs. 2011;15(6):593-595.

17. Henry DH, Viswanathan HN, Elkin LP, Traina S, Wade S, Cella D. Symptoms and treatment burden associated with cancer treatment: results from a cross-sectional national survey in the US. Support Care Cancer. 2008;16(7):791-801.

18. Hoffman M, Ryan JL, Figurosa-Moseley CD, Jean-Pierre P, Morrow GR. Cancer-related fatigue: the scale of the problem. Oncologist. 2007; 12 Suppl 1:4-10.

19. Wanchai A, Armer JM, Steward BR. Nonpharmacologic supportive strategies to promote quality of life in patients experiencing cancerrelated fatigue: a systematic review. Clin J Oncol Nurs. 2010;15(2): 203-214.

20. Poirier P. The relationship of sick leave benefits, employment patterns, and individual characteristics to radiation therapy-related fatigue. Oncol Nurs Forum. 2006;33(3):593-601.

21. Mitchell AA, Beck SL, Hood LE, Moore K, Tanner ER. Putting evidence into practice: evidence-based interventions for fatigue during and following cancer and its treatment. Clin J Oncol Nurs. 2009;11(1): 99-113.

22. National Comprehensive Cancer Network. Cancer-related fatigue. Version 1 2013, 2012. Available from: http://www.nccn.org. Accessed March 6, 2013.

23. Piper BF. Measuring fatigue. In: Frank-Strombotg M, Olsen SJ, editors. Instruments for Clinical Health-Care Research, 3rd ed. Sudbury, MA: Jones and Bartlett; 2004.

24. Butt Z, Wagner LI, Beaumont MS, et al. Use of a single-item tool to detect clinically significant fatigue, pain, distress, and anorexia in ambulatory cancer patients. J Pain Symptom Manage. 2008;3(1):20-30.

25. Oh HS, Seo WS. Systematic review and meta-analysis of the correlates of cancer-related fatigue. Worldviews Evid Based Nurs. 2011;8(4): 191-201.

26. Cramp F, Byron-Daniel J. Exercise for the management of cancer-related fatigue in adults. Cochrane Database Syst Rev. 2012;11:CD006145.

27. Hwang CL, Yu CJ, Shih JY, Yang PC, Wu YT. Effects of exercise training on exercise capacity in patients with non-small cell lung cancer receiving targeted therapy. Support Care Cancer. 2012;20(12): 3169-3177.

28. McNeely ML, Parliament MB, Seikaly H, et al. Effect of exercise on upper extremity pain and dysfunction in hand and neck cancer survivors: a randomized controlled trial. Cancer. 2008;113(1):214-222. 
29. Segal RJ, Reid RD, Courneya KS, et al. Randomized controlled trial of resistance or aerobic exercise in men receiving radiation therapy for prostate cancer. J Clin Oncol. 2009;27(3):344-351.

30. Courneya KS, Friedenreich CM, Quinney HA, Fields AL, Jones LW, Fairey AS. A randomized trial of exercise and quality of life in colorectal cancer survivors. Eur J Cancer Care. 2003;12(4):347-357.

31. Peddle CJ, Au HJ, Courneya KS. Associations between exercise, quality of life, and fatigue in colorectal cancer survivors. Dis Colon Rectum. 2008;51(8):1242-1248.

32. Oncology Nursing Society. Updated ONS putting evidence into practice resources. Clin J Oncol Nurs. 2009;13(2):137-143.

33. Donovan M, Glacken N. The lived experience of patients receiving radiation therapy for head and neck cancer: a literature review. Int $J$ Palliat Nurs. 2012;18(9):448-455.

34. Harris DJ, Eilers J, Harriman A, Cashavelly BJ, Maxwell C. Putting evidence into practice: evidence-based interventions for the management of oral mucositis. Clin J Oncol Nurs. 2007;12(1):141-152.

35. Eilers J. Nursing interventions and supportive care for the prevention and treatment of oral mucositis associated with cancer treatment. Oncol Nurs Forum. 2004;31 Suppl 4:13-23.

36. Russo G, Haddad R, Posner M, Machtay M. Radiation treatment breaks and ulcerative mucositis in head and neck cancer. Oncologist. 2008;13(8):886-898.

37. Multinational Association of Supportive Care in Cancer. Summary of evidence-based clinical practice guidelines for care of patients with oral and gastrointestinal mucositis. 2005 update. Available from: http:// www.mascc.org/mc/page.do?sitePageId=88037. Accessed February 10, 2013.

38. Lambertz CK, Gruell J, Robenstein V, Mueller-Funaiole V, Cummings K, Knapp V. No SToPS: reducing treatment breaks during chemoradiation for head and neck cancer. Clin J Oncol Nurs. 2011; 14(5):585-593.

39. McGuire DB, Correa ME, Johnson J, Wienandts P. The role of basic oral care and good clinical practice principles in the management of oral mucositis. Support Care Cancer. 2006;14(6):541-547.

40. Rubenstein EB, Peterson DE, Schubert M, et al; Mucositis Study Section of the Multinational Association for Supportive Care in Cancer; International Society for Oral Oncology. Clinical practice guidelines for the prevention and treatment of cancer therapy-induced oral and gastrointestinal mucositis. Cancer. 2004;100 Suppl 9:2026-2046.

41. Cawley MM, Benson LM. Current trends in managing oral mucositis. Clin J Oncol Nurs. 2005;9(5):584-592.

42. Eilers J, Million R. Clinical update: prevention and management of oral mucositis in patients with cancer. Semin Oncol Nurs. 2011;27(4): e1-e16.

43. Ontario Cancer Symptom Management Collaborative. Cancer Care Ontario's symptom management guide-to-practice: Oral care. 2012. Available from: https://www.cancercare.on.ca/toolbox/symptools/. Accessed February 10, 2013.

44. Scully C, Sonis S, Diz PD. Oral mucositis. Oral Dis. 2006;12(3): 229-241.

45. Guggenheimer J, Moore PA. Xerostomia: etiology, recognition, and treatment. J Am Dent Assoc. 2003;134(1):61-69.

46. Chambers MS, Rosenthal DI, Weber IS. Radiation-induced xerostomia. Head Neck. 2007;29(1):58-63.

47. Braam PM, Roesink JM, Paaijmakers PJ, Busschers WB, Terhaard CH. Quality of life and salivary output in patients with head-and-neck cancer five years after radiotherapy. Radiat Oncol. 2007;2:3.

48. Mendenhall NP, Malyapa RS, Su Z, Yeung D, Mendenhall WM, Li Z. Proton therapy for head and neck cancer: rationale, potential indications, practical considerations, and current clinical evidence. Acta Oncol. 2011;50(6):763-771.
49. Hensley ML, Hagerty KL, Kewalramani T, et al. American Society of Clinical Oncology 2008 clinical practice guideline update: use of chemotherapy and radiation therapy protectants. J Clin Oncol. 2009; 27(1):127-145.

50. Marcu LG. The role of amifostine in the treatment of head and neck cancer with cisplatin-radiotherapy. Eur J Cancer Care. 2009;18(2): 116-123.

51. Bensinger W, Schubert M, Ang K, et al. NCCN task force report: prevention and management of mucositis in cancer care. J Natl Comp Canc Netw. 2008;6 Supp1 1:S1-S21.

52. Hegarty AM, Hodgson TA. Management of xerostomia and salivary hypofunction. Prog Palliat Care. 2008;16(1):21-30.

53. Dauncey J, Greedy J, Morgan K. Prevention and management of treatment-related oral mucositis. Cancer Nursing Practice. 2012; 11(6):23-28. Available from: http://cancernursingpractice.rcnpublishing. co.uk/archive/article-prevention-and-management-of-treatmentrelated-oral-mucositis. Accessed February 10, 2013.

54. National Cancer Institute. Radiation therapy and you, 2007. Available from http://www.cancer.gov/cancertopics/coping/radiation-therapyand-you. Accessed February 10, 2013.

55. Cho J, Chung W, Kang W, Choi SM, Cho CK, Son CG. Manual acupuncture improved quality of life in cancer patients with radiationinduced xerostomia. J Altern Complement Med. 2008;14(5):523-526.

56. O'Sullivan EM, Higginson IJ. Clinical effectiveness and safety of acupuncture in the treatment of irradiation-induced xerostomia in patients with head and neck cancer: a systematic review. Acupunct Med. 2010;28(4):191-199.

57. Meng A, Garcia MK, Hu C, et al. Randomized controlled trial of acupuncture for prevention of radiation-induced xerostomia among patients with nasopharyngeal carcinoma. Cancer. 2012;118(13): 3337-3344.

58. Gosselin TK, Gilliard L, Tinnen R. Assessing the need for a dietitian in radiation oncology. Clin J Oncol Nurs. 2008;12(5):781-787.

59. [No authors listed]. Evidence-based practice guidelines for nutritional management of patients receiving radiation therapy. Nutr Diet. 2008; 65 Suppl 1:S1-S120.

60. Houlihan NG, Inzeo D, Joyce M, Tyson LB. Symptom management of lung cancer. Clin J Oncol Nurs. 2004;8(6):645-652.

61. Pena-Greenberg E, Law E, Frankel Kelvin J. Radiation-induced esophagitis: evidence-based nursing guidelines for lung and esophageal cancer patients. Oncol Nurs Forum. 2008;35(3):498.

62. Giralt J, Regadera JP, Verges R, et al. Effects of probiotic Lactobacillus casei DN-114 001 in the prevention of radiation-induced diarrhea. Int J Radiat Oncol Biol Phys. 2008;71(4):1213-1219.

63. Visich KL, Yeo TP. The prophylactic use of probiotics in the prevention of radiation therapy-induced diarrhea. Clin J Oncol Nurs. 2010;14(4): 467-473.

64. Muehlbauer PM, Thorpe D, Davis A, Drabot R, Rawlings BL, Kiker E. Putting evidence into practice: evidence-based interventions to prevent, manage, and treat chemotherapy and radiation therapy-induced diarrhea. Clin J Oncol Nurs. 2009;13(3):336-341.

65. Hoffman FA, Heimbach JT, Sanders ME, Hibberd PL. Executive summary: scientific and regulatory challenges of development of probiotics as foods and drugs. Clin Infect Dis. 2008;46 Suppl 2: S53-S57.

66. Reid G, Jass J, Sebulsky MT, McCormick JK. Potential uses of probiotics in clinical practice. Clin Microbiol Rev. 2003;16(4):658-672.

67. Blanarova C, Galovicova A, Petrasova D. Use of probiotics for prevention of radiation-induced diarrhea. Bratisl Lek Listy. 2009;110(2): 98-104. 
Nursing: Research and Reviews

\section{Publish your work in this journal}

Nursing: Research and Reviews is an international, peer-reviewed, open access journal publishing original research, reports, reviews and commentaries on all aspects of nursing and patient care. These include patient education and counselling, ethics, management and organizational issues, diagnostics and prescribing, economics and

resource management, health outcomes, and improving patient safety in all settings. The manuscript management system is completely online and includes a very quick and fair peer-review system. Visit http://www.dovepress.com/testimonials.php to read real quotes from published authors.

Submit your manuscript here: http://www.dovepress.com/nursing-research-and-reviews-journal 